\title{
Characteristics of volatile flavor components in traditional fermented yak milk produced in different ecoregions of the Qinghai-Tibetan plateau
}

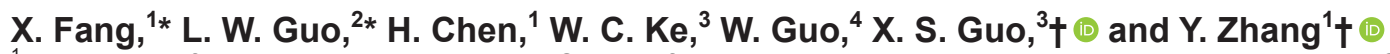 \\ ${ }^{1}$ Department of Nutrition and Food Hygiene, School of Public Health, Lanzhou University, Lanzhou 730000, PR China \\ 2Department of Epidemiology and Health Statistics, College of Public Health, Sichuan University, Chengdu 610041, PR China \\ ${ }^{3}$ State Key Laboratory of Grassland and Agro-Ecosystems, School of Life Sciences, Lanzhou University, Lanzhou 730000, PR China \\ ${ }^{4}$ College of Pastoral Agricultural Science and Technology, Lanzhou University, Lanzhou 730000, PR China
}

\begin{abstract}
The volatile flavor substances in traditional fermented yak milk samples collected from 5 ecoregions (A: coniferous forests and grasslands of the Qilian Qingdong Mountains; B: alpine grasslands surrounding the lakes in the Qiangtang Plateau; C: alpine shrubs and meadows of the Guoluo-Nagqu Highlands; D: coniferous forests along the alpine valley in East Tibet; E: shrubs and grasslands along the alpine valley in South Tibet) of the Qinghai-Tibetan plateau were comparatively analyzed. The relative percentage composition of volatile flavor substances varied among the different ecoregions. In samples collected from region $\mathrm{E}$, more than $50 \%$ of the volatile flavor compounds were esters comprising mainly $n$-butyl acetate, butyl butyrate, and ethyl octanoate, and a considerable proportion of acetoin was found in samples from regions B and E. Greater proportions of 2-heptanone and 2-nonanone were observed in samples collected from regions $\mathrm{A}, \mathrm{C}$, and D compared with regions B and E.

Key words: traditional fermented yak milk, volatile flavor component, yak, Qinghai-Tibetan plateau
\end{abstract}

\section{INTRODUCTION}

Traditional and naturally fermented dairy products, such as kefir, kumis, and ayran, are popular among consumers worldwide owing to their high nutritional value and unique flavors (Irigoyen et al., 2012; Laureys and Vuyst, 2014; Baruzzi et al., 2016). For thousands of years, local herdsmen living in the Qinghai-Tibetan plateau at altitudes above 4,000 $\mathrm{m}$ have been manufacturing traditional fermented yak milk called kurut.

Received July 23, 2019.

Accepted September 11, 2019.

*These authors contributed equally to this work.

†Corresponding authors: guoxsh07@lzu.edu.cn and yingz@lzu.edu .cn
This traditional product has higher TS, protein, fat, lactic acid, mineral (e.g., calcium, phosphorus, and magnesium), and vitamin $\mathrm{B}$ and $\mathrm{C}$ contents than cow milk-based yogurts (Zhang et al., 2008; Wu et al., 2009). In addition, traditional fermented yak milk has a high exopolysaccharide content from lactic acid bacteria (LAB), which contributes to its excellent curdling and probiotic effects (Laws and Marshall, 2001; Ding, 2014). The functional components of domestic yak (Bos grunniens) dairy products, especially traditional fermented yak milk, play important roles in maintaining the health of Tibetan herdsmen given the lack of fruits and vegetables in the extreme environments of the Tibetan plateau (Guo et al., 2014; Ding et al., 2017).

The palatability and consumer acceptability of fermented foods rely on the concentrations and types of volatile flavor substances, which serve as one of the important indicators for assessing fermented dairy products. It has been found that fermented dairy products contain more than 100 types of flavor substances, including carbonyl compounds, alcohols, acids, esters, hydrocarbons, aromatic compounds, and sulfur-containing compounds, all with varying odor and flavor threshold values (Caudle et al., 2005; Cheng, 2010; Vénica et al., 2016). Volatile flavor substances in yogurts are diverse compounds that are present in minute amounts with wide ranges of flavor thresholds $(0.001-1,000 \mu \mathrm{g} / \mathrm{L}$; Imhof et al., 1994). Previous studies have suggested that volatile substances, such as carbonyl compounds (e.g., acetaldehyde, diacetyl, and acetoin), ethanol, acetic acid, and ethyl acetoacetate, play important roles in the unique flavors of yogurts, but not all of the detected volatile substances contribute to yogurt flavor (Kneifel et al., 1992; Guzel-Seydim et al., 2000; Cheng, 2010). It has also been suggested that the unique flavors of yogurts are attributed not only to the diversity of volatile flavor substances but also to their percentage composition (Sidira et al., 2017).

Flavor substances in yogurts (e.g., acetaldehyde, diacetyl, acetoin) may be generated via microbial metabolic pathways (Cheng, 2010), and the Maillard 
reaction alters yogurt flavors through the production of flavor substances, such as sulfur-containing compounds and internal esters, via the heat treatment of dairy products during processing (van Boekel, 2006; Wit and Nieuwenhuijse, 2008). Numerous volatile flavor substances are derived from the microbial catabolism of proteins, lactose, and fatty acids in yogurts, where mixed starter cultures produce greater varieties of volatile flavor substances than single starter cultures (Beshkova et al., 1998). Besides this, traditional fermented yogurts have greater varieties and contents of volatile flavor substances than commercially produced yogurts (plain yogurt; Irigoyen et al., 2012). Naturally fermented dairy products are usually produced via the fermentation mediated by LAB and yeasts. The interaction between these 2 types of microorganisms affects the fermentation of dairy products (Rea et al., 1996; Viljoen, 2001), where the LAB are a key factor affecting the flavor and quality of the finished products. Natural fermentation using LAB may harbor more biologically active AA convertases that play key roles in the formation of AA-derived flavor substances, thereby exhibiting relatively greater effects and significance on flavor formation than in industrially manufactured fermented dairy products (Wouters et al., 2002).

To date, the majority of studies on traditional fermented yak milk from the Qinghai-Tibetan plateau have focused on its microbial diversity and probiotic role (Zhang et al., 2008; Wu et al., 2009; Ding et al., 2017), whereas its volatile flavor substances are rarely studied. Therefore, in this study we examined the compositions of volatile flavor substances in traditional fermented yak milk collected from 5 different ecoregions of the Qinghai-Tibetan plateau.

\section{MATERIALS AND METHODS}

\section{Sample Collection}

Traditional fermented yak milk samples were collected from 5 ecoregions of the Qinghai-Tibetan plateau (A: coniferous forests and grasslands of the Qilian Qingdong Mountains; B: alpine grasslands surrounding the lakes in the Qiangtang Plateau; C: alpine shrubs and meadows of the Guoluo-Nagqu Highlands; D: coniferous forests along the alpine valley in East Tibet; E: shrubs and grasslands along the alpine valley in South Tibet; Figure 1). These regions are the major areas of raising domestic yaks in the Qinghai-Tibetan plateau. The sampling sites covered 17 counties from elevations that ranged from 2,612 to $4,977 \mathrm{~m}$; latitudes ranged from $30^{\circ} 03^{\prime} \mathrm{N}$ to $37^{\circ} 52^{\prime} \mathrm{N}$, and longitudes ranged from $87^{\circ} 13^{\prime} \mathrm{E}$ to $102^{\circ} 56^{\prime} \mathrm{E}$. A total of 232 traditional fermented yak milk samples were collected from local herder households of the sampling sites, including 34 , $36,116,26$, and 20 samples from ecoregions A, B, C, $\mathrm{D}$, and $\mathrm{E}$, respectively. There were $3,3,7,4$, and 3 sampling counties included in ecoregions A, B, C, D, and E, respectively (Figure 1). All farmers adopted the same procedure for making traditional fermented yak milk, and the methodology was reported by Dong et al. (2003). Briefly, freshly boiled whole milk is poured into a pail. After the milk naturally cools to around $50^{\circ} \mathrm{C}$, a lump of household-preserved fermented yak milk (used as a fermentation starter) made during the last year is added, and the milk is mixed until the temperature has decreased to $40^{\circ} \mathrm{C}$. The pail is then covered and wrapped in wool to keep it warm; 5 or $6 \mathrm{~h}$ later in the warm season, and longer in winter, the milk will have soured and solidified. Traditional fermented yak milk samples were collected in sterile tubes $(15 \mathrm{~mL})$ and kept in a mini freezer $\left(-15^{\circ} \mathrm{C}\right)$ installed in a car. After transporting to the laboratory, the samples were stored at $-20^{\circ} \mathrm{C}$ for later analysis.

\section{Determination of Volatile Compounds}

Before pretreatment of the samples for analysis of volatile compounds, samples from each ecoregion were thawed at room temperature $\left(\sim 25^{\circ} \mathrm{C}\right)$. Two milliliters of each thawed sample from the same county (Table 1) was taken out and evenly mixed into several subsamples using a blender. Eventually, the 34, 36, 116, 26, and 20 samples from ecoregions A, B, C, D, and E were mixed into $3,3,7,4$, and 3 mixed subsamples based on the counties from each ecoregion (samples from each county were mixed into 1 subsample), respectively; these subsamples were then used for volatile compounds analysis. The volatile flavor components of the traditional fermented yak milk samples were extracted and determined as described previously (Erkaya and Sengul, 2011). Briefly, $10 \mathrm{~g}$ of each mixed subsample was transferred into a $50-\mathrm{mL}$ vial, and $3 \mathrm{~g}$ of $\mathrm{NaCl}$ and $100 \mu \mathrm{L}$ of $13 \mathrm{~m} M$ L-borneol (Sigma-Aldrich, St. Louis, MO) were added as an internal standard. Then, the rotor of a magnetic heated stirrer (Joan Lab, Ningbo City, China) was put in the vial, which was sealed. The prepared vials were kept at $40^{\circ} \mathrm{C}$ for $10 \mathrm{~min}$ in the thermostatic bath of the magnetic heated stirrer. For sampling, the solid-phase microextraction (SPME) device filled with carboxen-polydimethylsiloxane fiber $(75-\mu \mathrm{m}$ film thickness; Supelco, Bellefonte, PA) was inserted into the sealed vial containing the samples prepared as described above, and the fiber was exposed to the headspace for $40 \mathrm{~min}$ at $40^{\circ} \mathrm{C}$ during the headspace equilibration. Only the part of the vial with matrix was submerged in the thermostatic bath (Bianchi et al., 2007). 




Figure 1. Locations for collecting the traditional fermented yak milk samples from 5 ecoregions in the Qinghai-Tibetan plateau.

After sampling, a gas chromatograph-coupled mass selective detector (Perkin Elmer, Waltham, MA) was used to analyze the sample headspace components as described by Settachaimongkon et al. (2014). Briefly, the SPME device was immediately inserted into the
GC injector port, and the fiber was thermally desorbed at $250^{\circ} \mathrm{C}$ with a desorption time of $5 \mathrm{~min}$. The volatile flavor compounds were separated on a DB-FFAP quartz capillary column $(60 \mathrm{~m}, 0.25 \mathrm{~mm}$ i.d., $0.50-\mu \mathrm{m}$ film thickness; Agilent Technologies, Santa Clara, CA).

Table 1. Relative composition (\%) of volatile flavor compounds in traditional fermented yak milk produced in different ecoregions in the Qinghai-Tibetan Plateau

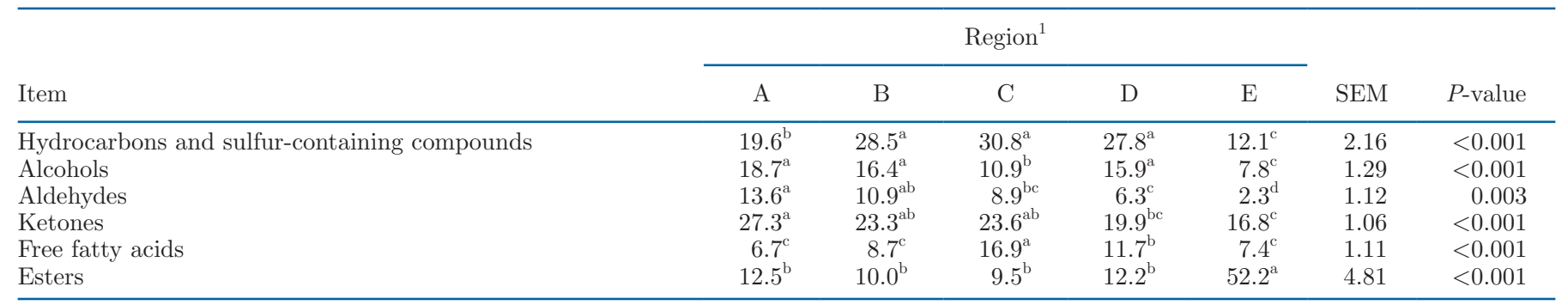

${ }^{\mathrm{a}-\mathrm{d}}$ Within a row, means with different superscripts differ $(P<0.05)$.

${ }^{1} \mathrm{~A}=$ coniferous forests and grasslands of the Qilian Qingdong Mountains; B = alpine grasslands surrounding the lakes in the Qiangtang Plateau; $\mathrm{C}=$ alpine shrubs and meadows of the Guoluo-Nagqu Highlands; $\mathrm{D}=$ coniferous forests along the alpine valley in East Tibet; $\mathrm{E}=$ shrubs and grasslands along the alpine valley in South Tibet. 
When the fiber was inserted, the temperature program was started and held at $40^{\circ} \mathrm{C}$ for $2 \mathrm{~min}$; then, it increased from $40^{\circ} \mathrm{C}$ to $200^{\circ} \mathrm{C}$ at $50^{\circ} \mathrm{C} / \mathrm{min}$ and from $200^{\circ} \mathrm{C}$ to $240^{\circ} \mathrm{C}$ at $10^{\circ} \mathrm{C} / \mathrm{min}$; finally, it was kept for another 2 min at $240^{\circ} \mathrm{C}$. The GC-MS interface was maintained at $250^{\circ} \mathrm{C}$. The carrier gas was helium with a constant flow rate of $1 \mathrm{~mL} / \mathrm{min}$, and the splitless injection was used. The MS ion source was maintained at $180^{\circ} \mathrm{C}$ with full scan. Electron impact mode was at $70 \mathrm{eV}$, with a mass range 35 to $400 \mathrm{~m} / z$ (mass:charge ratio). The peak area was identified by comparison of volatile experimental spectra from a database developed by the National Institute of Standards and Technology (NIST) and Wiley Online Library, and retention indices of authentic standard compounds were analyzed under identical conditions. Specific retention time and $\mathrm{m} / z$ model were used for automated peak integration in the XCalibur software package (Thermo Scientific, Austin, TX). The peak area:internal standard peak area ratio was taken as an arbitrary unit for estimating the quantities of each volatile compound. Peaks from column bleed and SPME fiber were corrected using the blank sample.

\section{Statistical Analysis}

Data were subjected to 1-way ANOVA using SPSS 19.0 (SPSS Inc., Chicago, IL), and Tukey's test was used to compare the differences among various groups. Principal component analysis was also performed using SPSS on the volatile flavor compounds [total hydrocarbons and sulfur-containing compounds, alcohols, aldehydes, ketones, free fatty acids (FFA), and esters of the mixed sample from the 5 ecoregions] to ascertain which of the attributes were most important for discriminating the samples. Differences were considered significant when the probability was less than 0.05 .

\section{RESULTS}

\section{Volatile Substances in Traditional Fermented Yak Milk from 5 Ecoregions of the Tibetan Plateau}

There were a wide variety of volatile substances in the traditional fermented yak milk samples collected from the 5 ecoregions of the Tibetan plateau, including hydrocarbons, sulfur-containing compounds, alcohols, aldehydes, ketones, FFA, and esters (Table 1). The relative contents of hydrocarbons, sulfur-containing compounds, alcohols, ketones, and esters varied significantly among the samples from the different ecoregions $(P<0.05)$. Among the 5 ecoregions, the traditional fermented yak milk collected from region $\mathrm{E}$ showed the lowest relative contents of hydrocarbons and sulfur- containing compounds, alcohols, and aldehydes $(P<$ 0.05). Samples from region $\mathrm{C}$ had the highest FFA, whereas esters in samples from region $\mathrm{E}$ accounted for more than half of the proportion of the total volatile flavor compounds.

The major hydrocarbons mainly included ethylbenzene, xylene, and styrene. The highest levels of ethylbenzene and styrene were observed in samples from region $\mathrm{C}$, whereas the lowest levels of xylene were found in samples from region $\mathrm{E}$ (Table 2). Only 1 sulfurcontaining component of dimethyl sulfide was detected, with the lowest value in samples from region $\mathrm{E}$. The alcoholic volatile flavor substances in traditional fermented yak milk mainly comprised 2-methyl-1-butanol, pentanol, heptanol, 2-ethylhexanol, 1-octanol, 1-nonanol, phenyl alcohol, and phenol, among which 2-methyl1-butanol and pentanol were the major alcohols (Table 3). Samples from region B had a predominant, large proportion of 2-methyl-1-butanol. There were 7 major aldehydes in the traditional fermented yak milk (Table 3 ). The highest levels of isovaleraldehyde and 3-methyl2-butenal were observed in samples from region $\mathrm{A}$, and samples from region $\mathrm{B}$ and $\mathrm{C}$ had the highest hexanal and octanal values, respectively. Ketones mainly comprised acetoin, 2-heptanone, and 2-nonanone (Table 4). Acetoin in samples from regions B and $\mathrm{E}$ was distinctly higher than that in the samples from other regions, and the highest 2-heptanone value was observed in samples from region $\mathrm{A}(P<0.05)$. For the aromatic component of acetone, the highest relative concentration was observed in samples from region $\mathrm{C}$, and samples from regions $\mathrm{A}$ and $\mathrm{C}$ had higher diacetyl levels than samples from the remaining regions $(P<0.05)$. The FFA in the traditional fermented yak milk mainly comprised acetic acid, 3-hydroxypropionic acid, butyric acid, hexanoic acid, and octanoic acid (Table 4). The highest levels of acetic acid, 3-hydroxypropionic acid, and octanoic acid were detected in the samples from region $\mathrm{C}(P<$ 0.05 ), and there was no difference in relative concentration of hexanoic acid in samples among the 5 regions $(P>0.05)$. For the esters in the traditional fermented yak milk, levels of $n$-butyl acetate, butyl butyrate, and ethyl octanoate in the samples from region $\mathrm{E}$ were dramatically higher than those from the other regions (Table 4). Moreover, the highest relative concentration of ethyl acetate was observed in samples from region A.

\section{Different Types of Volatile Flavor Substances in All of the Traditional Fermented Yak Milk Samples}

There were on average 63 to 77 types of volatile flavor substances in all of the traditional fermented yak milk samples (Table 5). There were no significant differences in the total variety of volatile flavor substances among 
Table 2. Major hydrocarbons and sulfur-containing compounds in traditional fermented yak milk sampled from different ecoregions in the Tibetan plateau

\begin{tabular}{|c|c|c|c|c|c|c|c|}
\hline Volatile compound $^{1}$ & \multicolumn{5}{|c|}{ Region $^{2}$} & SEM & $P$-value \\
\hline Methyl sulfide & $0.14^{\mathrm{ab}}$ & $0.20^{\mathrm{a}}$ & $0.16^{\mathrm{ab}}$ & $0.16^{\mathrm{ab}}$ & $0.08^{\mathrm{c}}$ & 0.012 & 0.034 \\
\hline 1,4-Pentadiene & $0.79^{\mathrm{a}}$ & $0.18^{\mathrm{bc}}$ & $0.28^{\mathrm{b}}$ & $0.10^{\mathrm{bc}}$ & $0.04^{\mathrm{c}}$ & 0.072 & $<0.001$ \\
\hline Hexane & 0.34 & 0.18 & 0.28 & 0.10 & 0.04 & 0.045 & 0.142 \\
\hline Pentane & 0.01 & 0.29 & 0.15 & 0.19 & 0.05 & 0.042 & 0.202 \\
\hline Benzene & $2.57^{\mathrm{a}}$ & $1.06^{\mathrm{b}}$ & $1.25^{\mathrm{b}}$ & $1.04^{\mathrm{c}}$ & $1.03^{\mathrm{c}}$ & 0.056 & $<0.001$ \\
\hline$p$-Xylene & $1.85^{\mathrm{ab}}$ & $2.09^{\mathrm{a}}$ & $2.19^{\mathrm{a}}$ & $1.92^{\mathrm{ab}}$ & $1.13^{\mathrm{c}}$ & 0.120 & 0.014 \\
\hline Styrene & $2.54^{\mathrm{d}}$ & $11.96^{\mathrm{b}}$ & $13.62^{\mathrm{a}}$ & $6.02^{\mathrm{c}}$ & $6.10^{\mathrm{c}}$ & 1.101 & 0.023 \\
\hline Imonene & 0.28 & 0.56 & 0.14 & 0.21 & 0.12 & 0.087 & 0.578 \\
\hline 1,2,4,5-Tetramethylbenzene & $0.28^{\mathrm{a}}$ & $0.14^{\mathrm{b}}$ & $0.24^{\mathrm{ab}}$ & $0.17^{\mathrm{ab}}$ & $0.04^{\mathrm{c}}$ & 0.024 & 0.001 \\
\hline 1-Methyl-4-methyl-benzene & $0.08^{\mathrm{ab}}$ & $0.25^{\mathrm{a}}$ & $0.12^{\mathrm{ab}}$ & $0.22^{\mathrm{ab}}$ & $0.03^{\mathrm{c}}$ & 0.027 & 0.030 \\
\hline Naphthalene & 1.34 & 1.45 & 0.89 & 1.08 & 1.27 & 0.147 & 0.155 \\
\hline 1-Methylnaphthalene & 0.39 & 0.18 & 0.37 & 0.21 & 0.22 & 0.065 & 0.278 \\
\hline
\end{tabular}

${ }^{\mathrm{a}-\mathrm{d}}$ Within a row, means with different superscripts differ $(P<0.05)$.

${ }^{1}$ The ratio peak area/IS peak area was used to calculate the concentration of each volatile component. Arbitrary units were used.

${ }^{2} \mathrm{~A}=$ coniferous forests and grasslands of the Qilian Qingdong Mountains; B = alpine grasslands surrounding the lakes in the Qiangtang Plateau; $\mathrm{C}=$ alpine shrubs and meadows of the Guoluo-Nagqu Highlands; $\mathrm{D}=$ coniferous forests along the alpine valley in East Tibet; $\mathrm{E}=$ shrubs and grasslands along the alpine valley in South Tibet.

the ecoregions, with the exception of the types of hydrocarbons and sulfur-containing compounds in the samples collected from region $\mathrm{A}$, which were lower than those from the other regions $(P<0.05)$. Among the flavor substances, hydrocarbons and sulfur-containing compounds had the greatest varieties (18-28 types), followed by the alcohols (13-16 types), aldehydes (9-11 types), ketones ( $8-10$ types), esters ( $7-10$ types), and FFA (4-6 types).

\section{Principal Component Analysis of the Composition of Volatile Flavor Substances in the Samples from 5 Ecoregions of the Tibetan Plateau}

In general, yogurt flavors are collectively attributed to multiple volatile flavor substances. The principal component analysis of the composition of volatile flavor substances in the samples collected from the different ecoregions is shown in Figure 2. The figure shows that

Table 3. Major alcohols and aldehydes in traditional fermented yak milk sampled from different ecoregions in the Tibetan plateau

\begin{tabular}{|c|c|c|c|c|c|c|c|}
\hline \multirow[b]{2}{*}{ Volatile compound $^{1}$} & \multicolumn{5}{|c|}{ Region $^{2}$} & \multirow[b]{2}{*}{ SEM } & \multirow[b]{2}{*}{$P$-value } \\
\hline & $\mathrm{A}$ & B & $\mathrm{C}$ & $\mathrm{D}$ & $\mathrm{E}$ & & \\
\hline 2-Methyl-1-butanol & $6.61^{\mathrm{b}}$ & $9.16^{\mathrm{a}}$ & $1.89^{\mathrm{d}}$ & $6.25^{\mathrm{b}}$ & $3.87^{\mathrm{c}}$ & 0.756 & 0.001 \\
\hline Pentanol & $0.57^{\mathrm{bc}}$ & $0.98^{\mathrm{b}}$ & $2.54^{\mathrm{a}}$ & $0.33^{\mathrm{c}}$ & $0.54^{\mathrm{bc}}$ & 0.131 & 0.003 \\
\hline 3-Pentanol & $3.48^{\mathrm{a}}$ & $2.39^{\mathrm{b}}$ & $2.25^{\mathrm{b}}$ & $0.45^{\mathrm{c}}$ & $0.25^{\mathrm{c}}$ & 0.151 & 0.001 \\
\hline Heptanol & $0.35^{\mathrm{a}}$ & $0.26^{\mathrm{ab}}$ & $0.19^{\mathrm{bc}}$ & $0.22^{\mathrm{bc}}$ & $0.13^{\mathrm{c}}$ & 0.023 & 0.017 \\
\hline 2-Ethylhexanol & $0.34^{\mathrm{a}}$ & $0.18^{\mathrm{ab}}$ & $0.19^{\mathrm{ab}}$ & $0.16^{\mathrm{b}}$ & $0.04^{\mathrm{b}}$ & 0.031 & 0.028 \\
\hline 1-Octanol & $1.97^{\mathrm{a}}$ & $0.21^{\mathrm{bc}}$ & $0.22^{\mathrm{bc}}$ & $0.32^{\mathrm{b}}$ & $0.11^{\mathrm{c}}$ & 0.084 & $<0.001$ \\
\hline 1-Nonanol & $2.79^{\mathrm{a}}$ & $0.64^{\mathrm{b}}$ & $0.46^{\mathrm{b}}$ & $0.53^{\mathrm{b}}$ & $0.15^{\mathrm{c}}$ & 0.161 & $<0.001$ \\
\hline Phenethyl alcohol & $0.38^{\mathrm{c}}$ & $1.17^{\mathrm{b}}$ & $0.52^{\mathrm{bc}}$ & $4.16^{\mathrm{a}}$ & $0.76^{\mathrm{bc}}$ & 0.187 & 0.001 \\
\hline Phenol & $0.34^{\mathrm{b}}$ & $0.37^{\mathrm{b}}$ & $0.47^{\mathrm{b}}$ & $0.96^{\mathrm{a}}$ & $0.11^{\mathrm{c}}$ & 0.076 & $<0.001$ \\
\hline Propanal & $0.28^{\mathrm{a}}$ & $0.12^{\mathrm{b}}$ & $0.16^{\mathrm{ab}}$ & $0.12^{\mathrm{b}}$ & $0.04^{\mathrm{b}}$ & 0.024 & 0.036 \\
\hline 2-Methylbutanal & 0.27 & 0.32 & 0.28 & 0.31 & 0.15 & 0.031 & 0.429 \\
\hline Isovaleraldehyde & $2.69^{\mathrm{a}}$ & $1.38^{\mathrm{b}}$ & $0.31^{\mathrm{c}}$ & $0.33^{\mathrm{c}}$ & $0.09^{\mathrm{d}}$ & 0.114 & $<0.001$ \\
\hline Hexanal & $1.83^{\mathrm{b}}$ & $3.23^{\mathrm{a}}$ & $0.15^{\mathrm{d}}$ & $1.07^{\mathrm{c}}$ & $0.38^{\mathrm{d}}$ & 0.292 & 0.017 \\
\hline 3-Methyl-2-butenal & $3.73^{\mathrm{a}}$ & $0.20^{\mathrm{c}}$ & $0.25^{\mathrm{c}}$ & $1.39^{\mathrm{b}}$ & $0.31^{\mathrm{c}}$ & 0.243 & 0.002 \\
\hline Benzaldehyde & $3.50^{\mathrm{a}}$ & $2.53^{\mathrm{b}}$ & $3.79^{\mathrm{a}}$ & $2.16^{\mathrm{b}}$ & $0.30^{\mathrm{c}}$ & 0.415 & 0.002 \\
\hline Octanal & $0.28^{\mathrm{c}}$ & $1.57^{\mathrm{b}}$ & $3.21^{\mathrm{a}}$ & $0.27^{\mathrm{c}}$ & $0.15^{\mathrm{c}}$ & 0.327 & $<0.001$ \\
\hline
\end{tabular}

${ }^{\mathrm{a}-\mathrm{d}}$ Within a row, means with different superscripts differ $(P<0.05)$.

${ }^{1}$ The ratio peak area/IS peak area was used to calculate the concentration of each volatile component. Arbitrary units were used.

${ }^{2} \mathrm{~A}=$ coniferous forests and grasslands of the Qilian Qingdong Mountains; B = alpine grasslands surrounding the lakes in the Qiangtang Plateau; $\mathrm{C}=$ alpine shrubs and meadows of the Guoluo-Nagqu Highlands; $\mathrm{D}=$ coniferous forests along the alpine valley in East Tibet; $\mathrm{E}=$ shrubs and grasslands along the alpine valley in South Tibet. 
Table 4. Major ketones, free fatty acids, and esters in traditional fermented yak milk sampled from different ecoregions in the Tibetan plateau

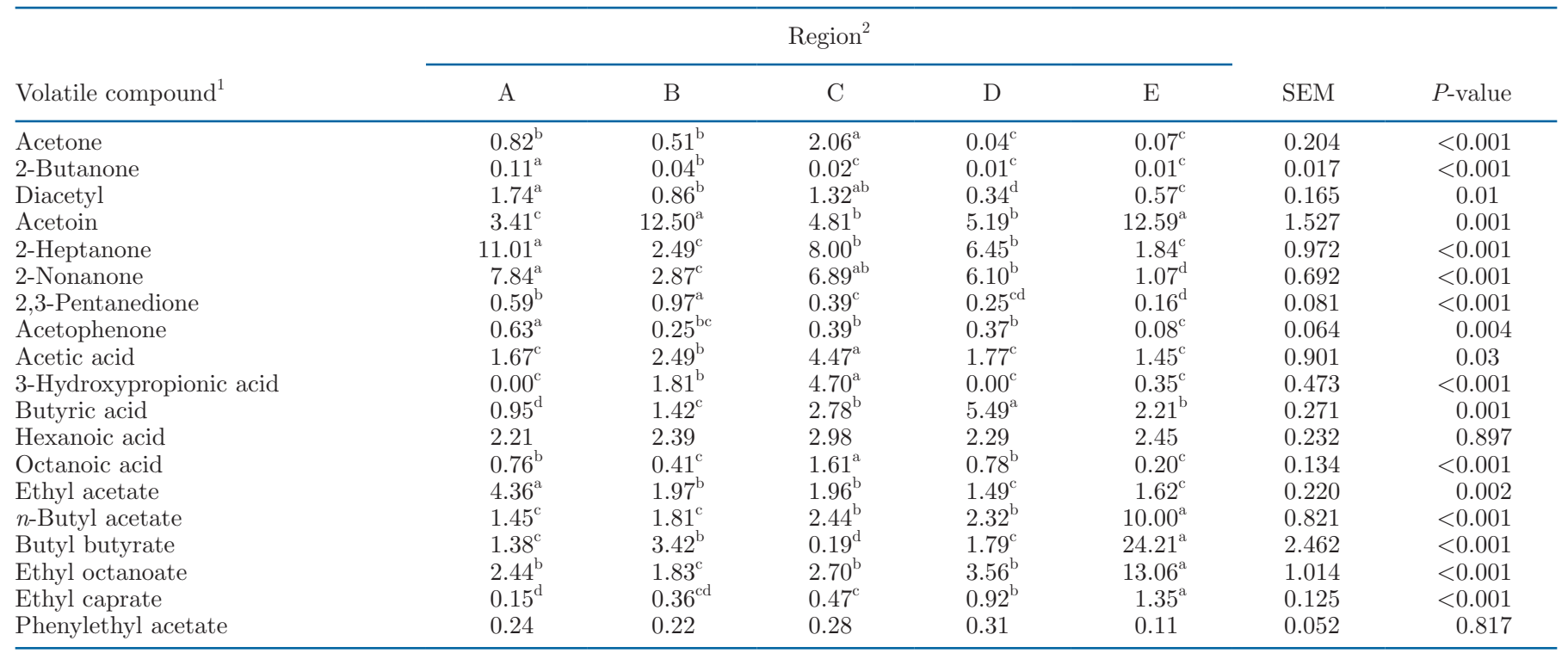

${ }^{\mathrm{a}-\mathrm{d}}$ Within a row, means with different superscripts differ $(P<0.05)$.

${ }^{1}$ The ratio peak area/IS peak area was used to calculate the concentration of each volatile component. Arbitrary units were used.

${ }^{2} \mathrm{~A}=$ coniferous forests and grasslands of the Qilian Qingdong Mountains; B = alpine grasslands surrounding the lakes in the Qiangtang Plateau; $\mathrm{C}=$ alpine shrubs and meadows of the Guoluo-Nagqu Highlands; $\mathrm{D}=$ coniferous forests along the alpine valley in East Tibet; $\mathrm{E}=$ shrubs and grasslands along the alpine valley in South Tibet.

the compositions of volatile flavor substances in the samples from regions $\mathrm{A}$ and $\mathrm{C}$ were similar to those in the samples from regions $\mathrm{B}$ and $\mathrm{D}$, respectively. On the contrary, the composition of volatile flavor substances in samples from region E were clearly different from that in samples from the other ecoregions. The FFA, alcohols, and aldehydes played important roles in contributing to the flavors of the traditional fermented yak milk from region $\mathrm{B}$, with relatively minor differences in their relative contents. In comparison, the relative contents of flavor substances had greater within-group variation. The relative contents of esters in the tra- ditional fermented yak milk from region E majorly contributed to the volatile flavor substances, with relatively minor within-group differences.

\section{DISCUSSION}

Traditional fermented yak milk is a unique yogurt from the Qinghai-Tibetan plateau (China) that has a delicate texture and distinct flavor. Our study showed that this traditional fermented product from the 5 ecoregions contained 63 to 77 types of volatile flavor substances (including aldehydes, ketones, esters,

Table 5. Number of types of volatile flavor components in traditional fermented yak milk produced in different ecoregions in the QinghaiTibetan Plateau

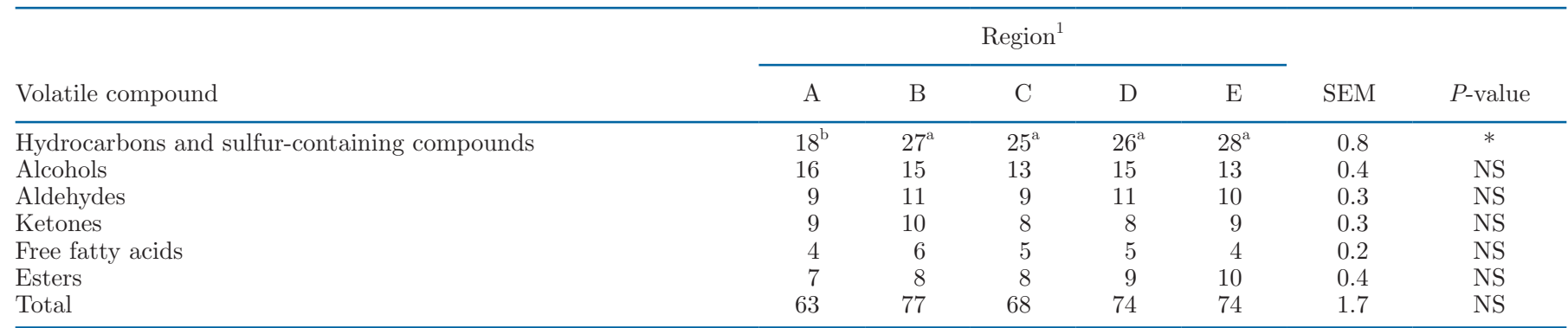

${ }^{\mathrm{a}, \mathrm{b}}$ Within a row, means with different superscripts differ $(P<0.05)$.

${ }^{1} \mathrm{~A}=$ coniferous forests and grasslands of the Qilian Qingdong Mountains; B = alpine grasslands surrounding the lakes in the Qiangtang Plateau; $\mathrm{C}=$ alpine shrubs and meadows of the Guoluo-Nagqu Highlands; $\mathrm{D}=$ coniferous forests along the alpine valley in East Tibet; $\mathrm{E}=$ shrubs and grasslands along the alpine valley in South Tibet.

$* P<0.05$. 


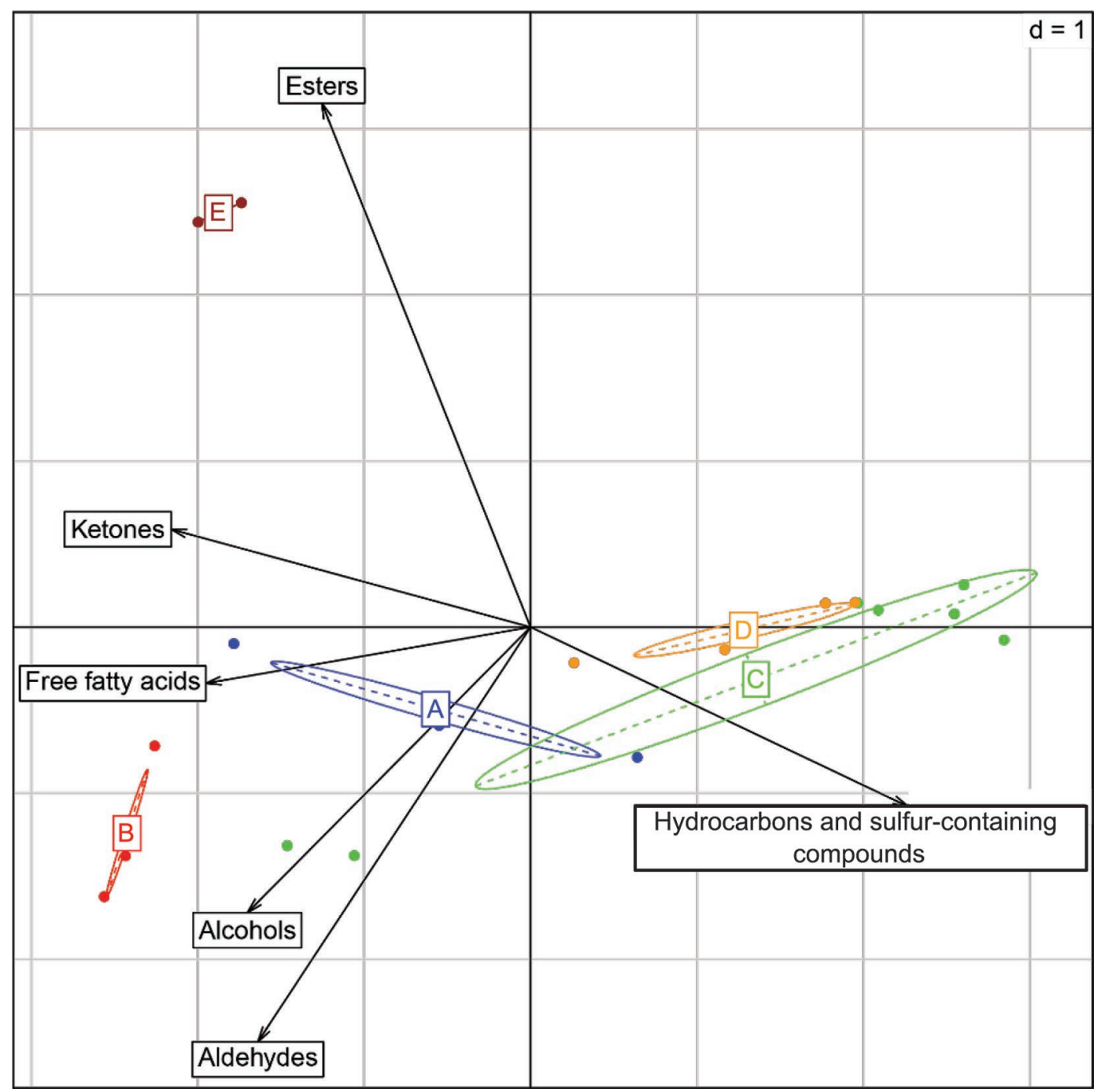

Figure 2. Principal component analysis of the relative percentage composition of volatile flavor compounds in traditional fermented yak milk produced in different ecoregions in the Qinghai-Tibetan Plateau. A = coniferous forests and grasslands of the Qilian Qingdong Mountains; $\mathrm{B}=$ alpine grasslands surrounding the lakes in the Qiangtang Plateau; $\mathrm{C}=$ alpine shrubs and meadows of the Guoluo-Nagqu Highlands; $\mathrm{D}=$ coniferous forests along the alpine valley in East Tibet; $\mathrm{E}=$ shrubs and grasslands along the alpine valley in South Tibet.

alcohols, FFA, hydrocarbons, and sulfur-containing compounds), which significantly outnumbered those in previously reported commercial yogurts (Irigoyen et al., 2012). Studies have indicated that using mixed-starter cultures results in greater varieties of flavor substances than does using single-starter cultures as well as more complicated microbial interactions (Beshkova et al., 1998). It has also been reported that multistrainfermented kefir contains up to 45 types of volatile flavor substances, which significantly outnumbers those in commercial fermented milk drinks and yogurt. Moreover, kefir is a fermented alcoholic dairy product with a greater variety of volatile alcoholic compounds (Irigoyen et al., 2012).
We found via principal component analysis that the composition of volatile flavor substances in traditional fermented yak milk varied among the different ecoregions and that each type of the volatile substances within the same ecoregion played varying roles. The traditional fermented yak milk samples collected from regions $\mathrm{B}, \mathrm{C}$, and $\mathrm{D}$ displayed the highest relative contents of hydrocarbons and dimethyl sulfide. A trace amount of volatile dimethyl sulfide is the main biological constituent contributing to the flavor and aroma of milk (Christensen and Reineccius, 1992). Dimethyl sulfide was detected in all the traditional fermented yak milk samples collected in this study, with a mean relative content of 0.08 to $0.20 \%$. Among the hydrocarbons, 
considerable relative contents of styrene and xylene were observed in the traditional fermented yak milk. These compounds might migrate from plastic packing materials (Nerín et al., 1998) because most of the traditional fermented yak milk samples were obtained from plastic pails in which the traditional fermented yak milk was made.

The traditional fermented yak milk samples collected from regions $\mathrm{A}, \mathrm{B}$, and $\mathrm{D}$ had the highest relative alcohol content, which played a relatively greater role in contributing to the traditional fermented yak milk flavors. Alcohols (comprising mostly 2-methyl-1-butanol and pentalnol) contribute to the mellow flavor of fermented milk and have a very high perception threshold (200 mg/L; Irigoyen et al., 2012). Although higher levels of ketones (comprising mostly acetone, acetoin, 2-heptanone, 2-nonanone, and diacetyl) were observed in samples collected from regions $\mathrm{A}$ and $\mathrm{C}$ compared with regions $\mathrm{D}$ and $\mathrm{E}$, considerably high levels of acetoin were found in regions B and E. In fermented milk products, acetoin is a common flavor compound that contributes to the sweet, fruity, mild, creamy, and butter-like flavor (Cheng, 2010; Wang and Arntfield, 2016). It was reported that 2-heptanone and 2-nonanone contributed to stale, cardboard, or metallic flavor in UHT milk (Moio et al., 1994). The relative higher levels of 2-heptanone and 2-nonanone in samples from regions $\mathrm{A}, \mathrm{C}$, and D suggested that the flavor of the traditional fermented yak milk from these regions was not as good as that from regions B and E, where values were lower. Esters (comprising mainly ethyl acetate, butyl acetate, butyl butyrate, and ethyl octanoate) constituted the highest relative content among the flavor substances in the traditional fermented yak milk collected from region E; they mainly contribute to the fruity (e.g., pineapple and banana) and aldehyde fragrances of yogurts (Cheng, 2010).

The composition and content of flavor substances in yogurts are affected by numerous factors, including the type of raw milk, milk processing, and fermentation agents (Cheng, 2010; Benozzi et al., 2015). The flavor substances in raw milk may be altered by forage grasses and seasonal changes (Dougherty et al., 1962). It has been demonstrated that different breeds of dairy cows produce varying qualities and flavors of milk, and high-fat and nutrient-rich milk has better quality and flavor (Guretzky et al., 2006). The total TA, protein, and fat concentrations in milk produced by domestic yak are almost twice as high as those in Holstein cattle milk (Guo et al., 2014). Therefore, the milk produced by domestic yak is also known as a natural condensed milk. It is an excellent raw material for manufacturing yogurts due to its mellow flavor. The catabolism of proteins, fats, and hydrocarbons in milk serves as one of the important sources of flavor substances in yogurts (Cheng, 2010). The types of forage grasses and feeding practices significantly affect the production of volatile flavor substances in milk. The continuous grazing system adopted by herdsmen on the QinghaiTibetan plateau provides domestic yaks with an abundant supply of forage grasses, thus ensuring the natural flavor of the animal's raw milk to a great extent. The flavor substances in forage grasses that enter the body of domestic yaks via digestion or respiration are metabolized and transported to the udder tissues through the bloodstream (Dougherty et al., 1962). However, the plateau vegetation cover varies among different ecoregions (from forests to shrubs, meadows, grasslands, and deserts) and deteriorates gradually from the southeast to northwest regions due to the harsher temperature and precipitation conditions (Zhang et al., 2010). To a certain extent, the variation of vegetation cover may affect the milk flavor of domestic yaks living in different ecoregions, which in turn affects the contents and types of volatile flavor substances present in traditional fermented yak milk.

Naturally fermented dairy products are typically manufactured via a mixed-culture fermentation process mediated by LAB and yeasts (Rea et al., 1996). Yeasts can either stimulate or inhibit the growth of LAB during the fermentation of dairy products. The direct or indirect interactions between these 2 types of microbes include competition, symbiosis, and antimicrobial effects. For instance, LAB provide a favorable environment for the growth of yeasts by secreting organic acids that reduce the $\mathrm{pH}$ of the fermented dairy products. Hence, the interaction between LAB and yeasts could improve the nutritional value and sensory characteristics of fermented dairy products (Viljoen, 2001). Zhang et al. (2008) studied the chemical and microbial compositions of traditional fermented yak milk and showed that it contained $9.18 \pm 0.81 \log _{10} \mathrm{cfu} / \mathrm{mL} \mathrm{LAB}$ and 8.33 $\pm 0.624 \log _{10} \mathrm{cfu} / \mathrm{mL}$ yeasts, which was significantly higher than levels in other types of yogurts. Additionally, the dominant microflora in traditional fermented yak milk varied among different regions, which may also affect the formation of volatile flavor substances in the product (data not shown).

Moreover, LAB is one of the most important factors affecting yogurt flavor and the quality of finished products. Previous studies have suggested that the content and types of flavor substances are associated with interspecies variations. It is generally believed that acetaldehyde is produced by Lactobacillus delbrueckii ssp. bulgaricus during fermentation (Neves et al., 2005). Subsequently, the acetaldehyde is converted 
into ethanol by alcohol dehydrogenases produced by Lactobacillus casei and Lactobacillus acidophilus (Ott et al., 2000). In this study, consistent with the results reported by $\mathrm{Wu}$ et al. (2009), acetaldehyde was not detected in any of the traditional fermented yak milk samples, probably due to its degradation by alcohol dehydrogenases in the yogurt. It has been shown that Streptococcus thermophilus and Leuconostoc citreum have relatively greater diacetyl-producing abilities (Iyer et al., 2010). Ding (2014) studied the LAB diversity in 232 traditional fermented yogurt samples collected from the 5 ecoregions described in this study and found that the main genera were Lactobacillus, Streptococcus, Enterococcus, Leuconostoc, Weissella, and Lactococcus. Lactobacillus and Streptococcus accounted for 98.5 to $99.7 \%$ of the total microbial relative abundance in the samples collected from all of these ecoregions, among which the relative abundance of Streptococcus in yogurts collected from regions A and E (44.6 and 50.1\%, respectively) was significantly higher than that in yogurts collected from regions $\mathrm{B}, \mathrm{C}$, and D $(37.4,10.5$, and $11.2 \%$, respectively). The present results indicated that hydrocarbons and sulfur-containing compounds had the highest relative contents in traditional fermented yak milk collected from regions B, C, and D. Meanwhile, esters had the highest relative content in traditional fermented yak milk collected from region E. These results indicated that Lactobacillus species play a major role in determining the content of hydrocarbons and sulfur-containing compounds in traditional fermented yak milk, whereas Streptococcus species play a decisive role in determining the content of esters. Additionally, the relationships of the volatile flavor substances in traditional fermented yak milk with the different types of grassland and yogurt microflora diversity need to be further explored.

\section{CONCLUSIONS}

There were on average 65 to 78 types of volatile flavor substances, including hydrocarbons, sulfur-containing compounds, alcohols, aldehydes, ketones, free AA, and esters, in the traditional fermented yak milk samples collected from 5 ecoregions in the Qinghai-Tibetan plateau. Although the relative contents of volatile flavor substances in the samples varied among the different ecoregions, the samples collected from geographically close ecoregions had similar flavor substance compositions. The relative concentrations of the main volatile flavor substances also varied among the different ecoregions. These variations might be related to the types of grassland and the composition of LAB in traditional fermented yak milk from the different regions, which need to be further studied.

\section{ACKNOWLEDGMENTS}

This research was supported by the National Natural Science Foundation of China, Beijing (31501976), the Fundamental Research Funds for the Central Universities of China, Lanzhou University (projects no. lzujbky-2018-70 and lzujbky-2017-k15), and the Innovation and Entrepreneurship Program of Lanzhou City, Lanzhou, China (2016-RC-55).

\section{REFERENCES}

Baruzzi, F., L. Quintieri, L. Caputo, P. Cocconcelli, M. Borcakli, L. Owczarek, U. T. Jasinska, S. Skapska, and M. Morea. 2016. Improvement of Ayran quality by the selection of autochthonous microbial cultures. Food Microbiol. 60:92-103.

Benozzi, E., A. Romano, V. Capozzi, S. Makhoul, L. Cappellin, L. Khomenko, E. Aprea, M. Scampicchio, G. Spano, T. D. Mark, F. Gasperi, and F. Biasioli. 2015. Monitoring of lactic fermentation driven by different starter cultures via direct injection mass spectrometric analysis of flavor-related volatile compounds. Food Res. Int. 76:682-688.

Beshkova, D., E. Simova, G. Frengova, and Z. Simov. 1998. Production of flavor compounds by yogurt starter cultures. J. Ind. Microbiol. Biotechnol. 20:180-186.

Bianchi, G., M. Nuzzi, A. A. Leva, and A. Rizzolo. 2007. Development of a headspace-solid phase micro extraction method to monitor changes in volatile profile of rose (Rosa hybrida, cv David Austin) petals during processing. J. Chromatogr. A 1150:190-197.

Caudle, A. D., Y. Yoon, and M. A. Drake. 2005. Influence of flavor variability in skim milk powder on consumer acceptability of ingredient applications. J. Food Sci. 70:S427-S431.

Cheng, H. 2010. Volatile flavor compounds in yogurt: A review. Crit. Rev. Food Sci. Nutr. 50:938-950.

Christensen, K. R., and G. A. Reineccius. 1992. Gas chromatographic analysis of volatile sulfur compounds from heated milk using static headspace sampling. J. Dairy Sci. 75:2098-2104.

Ding, W. R. 2014. Biodiversity and probiotic properties of lactic acid from spontaneously fermented yak milk in the Qinghai-Tibetan Plateau. PhD Thesis. Lanzhou University, Lanzhou, China. (In Chinese)

Ding, W. R., C. Shi, M. Chen, J. W. Zhou, R. J. Long, and X. S. Guo. 2017. Screening for lactic acid bacteria in traditional fermented Tibetan yak milk and evaluating their probiotic and cholesterollowering potentials in rats fed a high-cholesterol diet. J. Funct. Foods 32:324-332.

Dong, S. K., R. J. Long, and Y. M. Kang. 2003. Milking and milk processing: Traditional technologies in the yak farming system of the Qinghai-Tibetan plateau, China. Int. J. Dairy Technol. 56:86-93.

Dougherty, R. W., W. F. Shipe, G. V. Gudnason, and R. Scarpellino. 1962. Physiological mechanisms in transmitting flavors and odors to milk. J. Dairy Sci. 45:472-476.

Erkaya, T., and M. Sengul. 2011. Comparison of volatile compounds in yoghurts made from cows', buffaloes', ewes' and goats' milks. Int. J. Dairy Technol. 64:240-246.

Guo, X., R. J. Long, M. Kreuzer, L. M. Ding, Z. H. Shang, Y. Zhang, Y. Yang, and G. X. Cui. 2014. Importance of functional ingredients in yak milk-derived food on health of Tibetan nomads living under high-altitude stress: A review. Crit. Rev. Food Sci. Nutr. 54:292-302.

Guretzky, N. A. J., D. B. Carlson, J. E. Garrett, and J. K. Drackley. 2006. Lipid metabolite profiles and milk production for Holstein and Jersey cows fed rumen-protected choline during the periparturient period. J. Dairy Sci. 89:188-200.

Guzel-Seydim, Z. B., A. C. Seydim, A. K. Greene, and A. B. Bodine. 2000. Determination of organic acids and volatile flavor substances in Kefir during fermentation. J. Food Compos. Anal. 31:35-43. 
Imhof, R., H. Glattli, and J. O. Bosset. 1994. Volatile organic aroma compounds produced by thermophilic and mesophilic mixed strain dairy starter cultures. Lebensm. Wiss. Technol. 27:442-449.

Irigoyen, A., M. Ortigosa, S. Carcia, F. C. Ibanez, and P. Torre. 2012. Comparison of free amino acids and volatile components in three fermented milks. Int. J. Dairy Technol. 65:578-584.

Iyer, B., S. K. Tomar, T. U. Maheswari, and R. Singh. 2010. Streptococcus thermophilus strains: Multifunctional lactic acid bacteria. Int. Dairy J. 20:133-141.

Kneifel, W., M. Kaufmann, A. Fleischer, and F. Ulberth. 1992. Screening of commercially available mesophilic dairy starter cultures: Biochemical, sensory, and microbiological properties. J. Dairy Sci. 75:3158-3166.

Laureys, D., and L. D. Vuyst. 2014. Microbial species diversity, community dynamics, and metabolite kinetics of water kefir fermentation. Appl. Environ. Microbiol. 80:2564-2572.

Laws, A. P., and V. M. Marshall. 2001. The relevance of exopolysaccharides to the rheological properties in milk fermented with ropy strains of lactic acid bacteria. Int. Dairy J. 11:709-721.

Moio, L., P. Etievant, D. Langlois, J. Dekimpe, and F. Addeo. 1994. Detection of powerful odorants in heated milk by use of extract dilution sniffing analysis. J. Dairy Res. 61:385-394.

Nerín, C., C. Rubio, J. Cacho, and J. Salafranca. 1998. Parts pertrillion determination of styrene in yoghurt bypurge-and-trap gas chromatography with mass spectrometry detection. Food Addit. Contam. 15:346-354.

Neves, A. R., W. A. Pool, and J. Kok. 2005. Overview on sugar metabolism and its control in Lactococcus lactis - The input from in vivo NMR. FEMS Microbiol. Rev. 29:531-554.

Ott, A., A. Hugi, M. Baumgartner, and A. Chaintreau. 2000. Sensory investigation of yogurt flavor perception: Mutual influence of volatiles and acidity. J. Agric. Food Chem. 48:441-450.

Rea, M. C., T. Lennartsson, P. Dillon, F. D. Drinan, W. J. Reville, M. Heapes, and T. M. Cogan. 1996. Irish kefir-like grains: Their structure, microbial composition and fermentation kinetics. J. Appl. Bacteriol. 81:83-94.

Settachaimongkon, S., M. J. R. Nout, E. C. A. Fernandes, K. A. Hettinga, J. A. Vervoort, T. C. M. van Hooijdonk, M. H. Zwietering, E. J. Smid, and H. J. F. van Valenberg. 2014. Influence of different proteolytic strains of Streptococcus thermophilus in co-culture with Lactobacillus delbrueckii ssp. bulgaricus on the metabolite profile of set-yoghurt. Int. J. Food Microbiol. 177:29-36.
Sidira, M., V. Santarmaki, M. Kiourtzidis, A. A. Argyri, O. S. Papadopoulou, N. Chorianopoulos, C. Tassou, S. Kaloutsas, A. Galanis, and Y. Kourkoutas. 2017. Evaluation of immobilized Lactobacillus plantarum 2035 on whey protein as adjunct probiotic culture in yoghurt production. Lebensm. Wiss. Technol. 75:137-146.

van Boekel, M. A. 2006. Formation of flavour compounds in the Maillard reaction. Biotechnol. Adv. 24:230-233.

Vénica, C. I., I. V. Wolf, C. V. Bergamini, and M. C. Perotti. 2016. Influence of lactose hydrolysis on galacto-oligosaccharides, lactose, volatile profile and physicochemical parameters of different yogurt varieties. J. Sci. Food Agric. 96:4929-4939.

Viljoen, B. C. 2001. The interaction between yeasts and bacteria in dairy environments. Int. J. Food Microbiol. 69:37-44.

Wang, K., and S. D. Arntfield. 2016. Probing the molecular forces involved in binding of selected volatile flavour compounds to saltextracted pea proteins. Food Chem. 211:235-242.

Wit, R. D., and H. Nieuwenhuijse. 2008. Kinetic modelling of the formation of sulphur-containing flavour components during heattreatment of milk. Int. Dairy J. 18:539-547.

Wouters, J. T. M., E. H. E. Ayad, J. Hugenholtz, and G. Smit. 2002. Microbes from raw milk for fermented dairy products. Int. Dairy J. 12:91-109.

Wu, X. H., Z. Luo, L. Yu, F. Z. Ren, B. Z. Han, and M. J. Robert Nout. 2009. A survey on composition and microbiota of fresh and fermented yak milk at different Tibetan altitudes. Dairy Sci. Technol. 89:201-209.

Zhang, G. L., H. Ouyang, X. Z. Zhang, C. P. Zhou, and X. L. Xu. 2010. Vegetation change and its responses to climatic variation based on eco-geographical regions of Tibetan plateau. Geogr. Res. 11:2004-2016.

Zhang, H. P., J. Xu, J. G. Wang, Menghebilige, T. S. Sun, H. P. Li, and M. R. Guo. 2008. A survey on chemical and microbiological composition of traditional fermented yak milk, naturally fermented yak milk from Qinghai in China. Food Control 19:578-586.

\section{ORCIDS}

X. S. Guo ๑ https://orcid.org/0000-0002-5587-3920

Y. Zhang () https://orcid.org/0000-0003-1116-8290 